\title{
ON AN IMPROVEMENT IN CONFIDENCE LIMITS FOR PROPORTION OF CONFORMANCE
}

\author{
Michael Perakis and Evdokia Xekalaki \\ Department of Statistics \\ Athens University of Economics and Business \\ 76 Patision St., 104 34, Athens, GREECE
}

\begin{abstract}
This paper presents a modification of a method for constructing approximate lower confidence limits for the proportion of conformance of normally distributed processes, which was proposed by Wang and Lam (1996). The proposed method is very simple and its implementation does not require the use of special tables. In addition, as verified via simulation, it leads to confidence limits with coverage much closer to the nominal in comparison to the method of Wang and Lam (1996).
\end{abstract}

Keywords and Phrases: proportion of conformance; process capability indices; confidence limits; normal distribution; simulation.

\section{Introduction}

The proportion of conformance of a process is defined as the probability of producing within some given specifications. These specifications are determined through the lower specification limit (L) and the upper specification limit (U). Thus, if we denote the process by $\mathrm{X}$, its proportion of conformance is defined as

$$
\mathrm{p}=\mathrm{P}(\mathrm{L}<\mathrm{X}<\mathrm{U}) .
$$

The proportion of conformance plays an important role in Statistical Quality Control, since it is related, more or less directly, to the majority of the proposed process capability indices (see, e.g. Kotz and Johnson (1993); Kotz and Lovelace (1998) and Kotz and Johnson (2001)). On the other hand, even as a single measure, it 
provides considerable information on the capability of the process. For this reason, many authors have dealt with its estimation.

In the next section, a brief description of the three most widely used methods for constructing lower confidence limits (lcls) for the proportion of conformance is given, under the assumption that the examined process follows the normal distribution. A modification of one of these methods is considered in Section 3. It is shown via an extensive simulation study that this modification achieves a much better coverage. Finally, in Section 4, the use of the lower confidence limit suggested is illustrated on an example given by Wang and Lam (1996) in relation to their method.

\section{Some Methods for Assessing Lower Confidence Limits for the Proportion of Conformance}

Having assumed that the examined process, is normally distributed, the proportion of conformance is given by

$$
\mathrm{p}=\Phi\left(\frac{\mathrm{U}-\mu}{\sigma}\right)-\Phi\left(\frac{\mathrm{L}-\mu}{\sigma}\right),
$$

where $\mu, \sigma$ denote the mean and the standard deviation of the process, respectively and $\Phi($.$) is the cumulative distribution function of the standard normal distribution. In$ most of the cases the parameters $\mu$ and $\sigma$ are unknown and thus they have to be estimated in order to obtain an estimate of the proportion of conformance. A point estimator of $\mathrm{p}$ can be obtained by substituting in (1) the sample mean $(\bar{X})$ and the sample standard deviation (S) of a random sample taken from the process, for $\mu$ and $\sigma$, respectively. The resulting estimator of $\mathrm{p}$ is

$$
\hat{\mathrm{p}}=\Phi\left(\frac{\mathrm{U}-\overline{\mathrm{X}}}{\mathrm{S}}\right)-\Phi\left(\frac{\mathrm{L}-\overline{\mathrm{X}}}{\mathrm{S}}\right) .
$$

It should be remarked that the estimator of $\mathrm{p}$ given in (2) differs from its minimum variance unbiased estimator, but as Kotz and Johnson (1993) point out, it is much more convenient than the minimum variance unbiased estimator of $\mathrm{p}$ and it is quite adequate for most occasions.

The use of $\hat{\mathrm{p}}$ leads solely to a point estimator of $\mathrm{p}$. Nonetheless, a point estimator is not enough without having a corresponding confidence limit, which 
provides information on the accuracy of the estimation. For this reason, the construction of confidence limits for the true value of $\mathrm{p}$ is a very interesting problem that motivated many authors to deal with it. In the remaining of this section, three methods that enable one to construct approximate lcls for $\mathrm{p}$, are briefly described. The first is based on a method proposed by Owen and Hua (1977), the second is based on a method introduced by Chou and Owen (1984), while the third was developed by Wang and Lam (1996).

We commence with the method of Owen and Hua (1977). These authors sought confidence limits for probabilities $\mathrm{p}_{1}=\mathrm{P}(\mathrm{X}<\mathrm{L})$ and $\mathrm{p}_{2}=\mathrm{P}(\mathrm{X}>\mathrm{U})$ (apparently, $\left.\mathrm{p}=1-\mathrm{p}_{1}-\mathrm{p}_{2}\right)$. The assessment of such limits is possible by solving a complicated equation, which involves the non-central $t$ distribution. Let us denote by $\mathrm{p}_{1}^{\prime}$ and $\mathrm{p}_{2}^{\prime}$ the upper confidence limits for $\mathrm{p}_{1}$ and $\mathrm{p}_{2}$, respectively. Then, these values can be obtained for various sample sizes and confidence levels through the use of some tables provided by Owen and Hua (1977). As Wang and Lam (1996) point out, these tables can also be used for obtaining lcls for $\mathrm{p}$. In fact, an approximate lcl for $\mathrm{p}$ is given by

$$
1-\mathrm{p}_{1}^{\prime}-\mathrm{p}_{2}^{\prime}
$$

The second method considered in this section was proposed by Chou and Owen (1984). This method differs from that of Owen and Hua (1977), since it results in onesided simultaneous confidence regions on the upper and the lower tail areas of the normal distribution. If $p_{1}^{*}$ and $p_{2}^{*}$ are simultaneous upper confidence limits for $p_{1}$ and $\mathrm{p}_{2}$ they must satisfy the equation

$$
\mathrm{P}\left(\mathrm{p}_{1} \leq \mathrm{p}_{1}^{*} \text { and } \mathrm{p}_{2} \leq \mathrm{p}_{2}^{*}\right)=1-\alpha .
$$

Chou and Owen (1984) have tabulated the values of $p_{1}^{*}$ and $p_{2}^{*}$ for $\alpha=0.1,0.05,0.01$ and various sample sizes. These values can be used for the construction of lcls for the actual proportion of conformance of the process. In fact, a $100(1-\alpha) \%$ lcl for $p$ is a value $\mathrm{p}^{*}$ such that in $100(1-\alpha) \%$ of the cases the inequality

$$
\mathrm{p}=1-\mathrm{p}_{1}-\mathrm{p}_{2} \geq \mathrm{p}^{*}
$$

is satisfied. Having found the simultaneous upper confidence limits for $\mathrm{p}_{1}$ and $\mathrm{p}_{2}$, i.e. $\mathrm{p}_{1}^{*}$ and $\mathrm{p}_{2}^{*}$, one may deduce that in $100(1-\alpha) \%$ of the cases $\mathrm{p}_{1}+\mathrm{p}_{2} \leq \mathrm{p}_{1}^{*}+\mathrm{p}_{2}^{*}$, or equivalently 


$$
\mathrm{p} \geq 1-\mathrm{p}_{1}^{*}-\mathrm{p}_{2}^{*} .
$$

Hence, as Wang and Lam (1996) point out, a 100(1- $\alpha) \%$ lcl for the proportion of conformance is given by

$$
1-\mathrm{p}_{1}^{*}-\mathrm{p}_{2}^{*}
$$

An obvious drawback of the two methods that have been presented so far is that their implementation requires values provided in tables that are included in the corresponding articles. This limits their use. For this reason, Wang and Lam (1996) introduced another method for the construction of confidence limits for $p$, that is much simpler and does not require the availability of special tables. As clarified in the sequel, this method merely requires tables of the chi-square distribution. In particular, Wang and Lam (1996) sought a value $\mathrm{p}^{*}$ such that $\mathrm{P}\left(\mathrm{p} \geq \mathrm{p}^{*}\right)=1-\alpha$. Substituting $\mathrm{P}(\mathrm{L}<\mathrm{X}<\mathrm{U})$ for $\mathrm{p}$ they obtained

$$
\mathrm{P}\left[\mathrm{P}\left(\overline{\mathrm{X}}-\mathrm{K}_{1} \mathrm{~S}<\mathrm{X}<\overline{\mathrm{X}}+\mathrm{K}_{2} \mathrm{~S}\right) \geq \mathrm{p}^{*}\right]=1-\alpha,
$$

where $\mathrm{K}_{1}=(\overline{\mathrm{X}}-\mathrm{L}) / \mathrm{S}$ and $\mathrm{K}_{2}=(\mathrm{U}-\overline{\mathrm{X}}) / \mathrm{S}$. A standardization of the terms, so as to result in the standard normal distribution, leads to the equivalent form

$$
\mathrm{P}\left[\mathrm{P}\left(\frac{\overline{\mathrm{X}}-\mu}{\sigma}-\frac{\mathrm{K}_{1} \mathrm{~S}}{\sigma}<\frac{\mathrm{X}-\mu}{\sigma}<\frac{\overline{\mathrm{X}}-\mu}{\sigma}+\frac{\mathrm{K}_{2} \mathrm{~S}}{\sigma}\right) \geq \mathrm{p}^{*}\right]=1-\alpha .
$$

Under the assumption of normality of the process, the random variable $(\mathrm{X}-\mu) / \sigma$ follows the standard normal distribution and the left hand side of (5) reduces to

$$
\mathrm{P}\left[\Phi\left(\frac{\mathrm{Z}}{\sqrt{\mathrm{n}}}+\mathrm{K}_{2} \sqrt{\frac{\mathrm{Y}}{\mathrm{n}-1}}\right)-\Phi\left(\frac{\mathrm{Z}}{\sqrt{\mathrm{n}}}-\mathrm{K}_{1} \sqrt{\frac{\mathrm{Y}}{\mathrm{n}-1}}\right) \geq \mathrm{p}^{*}\right],
$$

where the random variable $\mathrm{Z}=\sqrt{\mathrm{n}}(\overline{\mathrm{X}}-\mu) / \sigma$ follows the standard normal distribution and the random variable $\mathrm{Y}=(\mathrm{n}-1) \mathrm{S}^{2} / \sigma^{2}$ follows the chi-square distribution with $\mathrm{n}-1$ degrees of freedom (df). Wang and Lam (1996) based on an earlier result described by Wald and Wolfowitz (1946) showed that if the probabilities that $\mathrm{K}_{1}$ and $\mathrm{K}_{2}$ are negative are very small, (6) can be approximated rather accurately by

$$
\mathrm{P}\left[\Phi\left(\frac{1}{\sqrt{\mathrm{n}}}+\mathrm{K}_{2} \sqrt{\frac{\mathrm{Y}}{\mathrm{n}-1}}\right)-\Phi\left(\frac{1}{\sqrt{\mathrm{n}}}-\mathrm{K}_{1} \sqrt{\frac{\mathrm{Y}}{\mathrm{n}-1}}\right) \geq \mathrm{p}^{*}\right] .
$$

As a consequence of the fact that $\mathrm{Y}$ follows the chi-square distribution with $\mathrm{n}-1$ df, they obtained a $100(1-\alpha) \%$ lcl for $p$ of the form 


$$
\mathrm{p}^{*}=\Phi\left(\frac{1}{\sqrt{\mathrm{n}}}+\max \left(\mathrm{K}_{1}, \mathrm{~K}_{2}\right) \sqrt{\frac{\chi_{\mathrm{n}-1, \alpha}^{2}}{\mathrm{n}-1}}\right)-\Phi\left(\frac{1}{\sqrt{\mathrm{n}}}-\min \left(\mathrm{K}_{1}, \mathrm{~K}_{2}\right) \sqrt{\frac{\chi_{\mathrm{n}-1, \alpha}^{2}}{\mathrm{n}-1}}\right),
$$

where $\chi_{n-1, \alpha}^{2}$ denotes the $\alpha$ quantile of the chi-square distribution with $n-1 \mathrm{df}$.

Wang and Lam (1996) conducted a simulation study in order to compare the performance of lcls (3), (4) and (7). They deduced that all the intervals are successful in maintaining the stated confidence level, even though they seem to be conservative (especially lcls (4) and (7)). Moreover, they deduced that if the tables that are essential for the assessment of lcls (3) and (4) are available, then lcl (3) is preferable to (4). On the other hand, if these tables are not available, lcl (7) can be used since its computation is fairly simple and its coverage, though higher than the nominal in all cases, is relatively satisfactory (only in a few cases results in coverage better than those of lcls (3) and (4)).

\section{A Modification of the Method by Wang and Lam (1996) - A Simulation Study}

In the previous section, three methods for assessing lcls for $\mathrm{p}$ were illustrated. However, the assessment of lcls (3) and (4) is very cumbersome since it requires the use of special tables, while lcl (7), though simple in its assessment, leads to conservative lcls. Hence, seeking for a lower confidence limit that would exhibit a coverage closer to the nominal would be of practical value.

In the sequel, a modification of Wang and Lam's lcl is proposed which, as shown via simulation, attains a coverage that is much closer to the nominal for almost all parameter combinations, sample sizes and significance levels considered while, at the same time, it retains the simplicity of $1 \mathrm{cl}$ (7). In particular, the following variant of (7) is proposed

$$
\Phi\left(\frac{1}{\sqrt{\mathrm{n}}}+\left(1+\mathrm{A}_{\mathrm{n}}\right) \max \left(\mathrm{K}_{1}, \mathrm{~K}_{2}\right) \sqrt{\frac{\chi_{\mathrm{n}-1, \alpha}^{2}}{\mathrm{n}-1}}\right)-\Phi\left(\frac{1}{\sqrt{\mathrm{n}}}-\left(1+\mathrm{A}_{\mathrm{n}}\right) \min \left(\mathrm{K}_{1}, \mathrm{~K}_{2}\right) \sqrt{\frac{\chi_{\mathrm{n}-1, \alpha}^{2}}{\mathrm{n}-1}}\right),
$$

where $A_{n}$ is a constant that depends on the sample size and tends to 0 as $n$ increases. The constant $1+\mathrm{A}_{\mathrm{n}}$ can play the role of a "correction" factor which can adjust the value of $\mathrm{p}^{*}$ as given by (7) to a less conservative one. The lcl for $\mathrm{p}$ defined by (8) is 
similar in nature to (7), but leads to a greater value of $\mathrm{p}^{*}$. This is a consequence of the fact that $\Phi($.$) is a non-decreasing function.$

For large values of the sample size, whence $A_{n}$ gets very close to 0 , the value of $p^{*}$ obtained by (8) is approximately equal to that obtained by (7). The choice of $A_{n}$ as a decreasing function of $\mathrm{n}$ was largely based on the fact that, as revealed by the results of a simulation study conducted by Wang and Lam (1996) and confirmed by our simulation results, in the majority of the cases (especially when the values of $\mathrm{k}_{1}=(\mu-\mathrm{L}) / \sigma$ and $\mathrm{k}_{2}=(\mathrm{U}-\mu) / \sigma$ are equal) the performance of (7) is improved as the sample size increases. So, the degree of the adjustment applied to $\mathrm{p}^{*}$ through a formula of type (8) lessens as the sample size gets larger.

The effect of the adjustment on $\mathrm{p}^{*}$ was examined for $\mathrm{A}_{\mathrm{n}}$ of the form $\mathrm{cn}^{-\mathrm{b}}$ for various values of $c$ and $b$. It was found via simulation that the form that had the best performance for a wider variety of parameter combinations was $A_{n}=1 / n$.

Taking account of the above considerations, the following modification of (7) is suggested:

$$
\ddot{\mathrm{p}}=\Phi\left(\frac{1}{\sqrt{\mathrm{n}}}+\mathrm{C}_{1}\right)-\Phi\left(\frac{1}{\sqrt{\mathrm{n}}}-\mathrm{C}_{2}\right),
$$

where

$$
\mathrm{C}_{1}=\left(1+\frac{1}{\mathrm{n}}\right) \max \left(\mathrm{K}_{1}, \mathrm{~K}_{2}\right) \sqrt{\frac{\chi_{\mathrm{n}-1, \alpha}^{2}}{\mathrm{n}-1}}
$$

and

$$
\mathrm{C}_{2}=\left(1+\frac{1}{\mathrm{n}}\right) \min \left(\mathrm{K}_{1}, \mathrm{~K}_{2}\right) \sqrt{\frac{\chi_{\mathrm{n}-1, \alpha}^{2}}{\mathrm{n}-1}} .
$$

In the sequel, a comparison of lcls (7) and (9) is made via a simulation study that we conducted. Random samples of various sizes were generated from the normal distribution, for various parameter combinations. For every combination of parameters and sample size, the total number of the generated samples was 25000 . For each of these 25000 samples, lcls for $p$ were assessed using formulae (7) and (9) and the percentage of times at which the actual value of the proportion of conformance exceeded each lcl was calculated. The sample sizes selected were $n=10$, 30,50 and 100 . It was assumed that $\mathrm{L}=10$ and $\mathrm{U}=20$ and various combinations of $\mathrm{k}_{1}=(\mu-\mathrm{L}) / \sigma$ and $\mathrm{k}_{2}=(\mathrm{U}-\mu) / \sigma$ were chosen (these combinations coincide to 
those considered in the simulation study conducted by Wang and Lam (1996)). Due to the symmetry of the normal distribution, processes with mean greater than 15 (i.e. $\mathrm{k}_{1}>\mathrm{k}_{2}$ ) have not been examined. The parameters of the examined processes are summarized in Table 1.

Table 1. The processes considered in the simulation study

\begin{tabular}{||c|c|c|c||c|c|c|c||}
\hline \hline $\mathbf{k}_{\mathbf{1}}$ & $\mathbf{k}_{\mathbf{2}}$ & $\boldsymbol{\mu}$ & $\boldsymbol{\sigma}$ & $\mathbf{k}_{\mathbf{1}}$ & $\mathbf{k}_{\mathbf{2}}$ & $\boldsymbol{\mu}$ & $\boldsymbol{\sigma}$ \\
\hline 1 & 1 & 15 & 5 & 3 & 3 & 15 & 1.667 \\
\hline 1 & 2 & 13.333 & 3.333 & 3 & 4 & 14.285 & 1.428 \\
\hline 1 & 3 & 12.5 & 2.5 & 3 & 5 & 13.75 & 1.25 \\
\hline 1 & 4 & 12 & 2 & 3 & 6 & 13.333 & 1.111 \\
\hline 1 & 5 & 11.666 & 1.667 & 4 & 4 & 15 & 1.25 \\
\hline 1 & 6 & 11.428 & 1.428 & 4 & 5 & 14.444 & 1.111 \\
\hline 2 & 2 & 15 & 2.5 & 4 & 6 & 14 & 1 \\
\hline 2 & 3 & 14 & 2 & 5 & 5 & 15 & 1 \\
\hline 2 & 4 & 13.333 & 1.667 & 5 & 6 & 14.545 & .909 \\
\hline 2 & 5 & 12.857 & 1.428 & 6 & 6 & 15 & .833 \\
\hline 2 & 6 & 12.5 & 1.25 & & & & \\
\hline
\end{tabular}

The results of the study are summarized in Tables 2 and 3. The top value in the entries of these tables corresponds to the observed coverage of the lcl given by (7), while the bottom values correspond to that of $1 \mathrm{cl}(9)$. Table 2 refers to the observed coverage for confidence level $1-\alpha=0.90$, while Table 3 refers to the observed coverage for confidence level 0.95 . One may observe that in all the cases, the observed coverage of $1 \mathrm{cl}(7)$ is greater than the nominal $1-\alpha$, something that confirms the results of Wang and Lam (1996), who asserted that lcl (7) is conservative. On the other hand, the coverage of (9) is much closer to the nominal in almost all the cases, for every sample size, confidence level and parameters combination. Likewise, even though the coverage of (9) is usually greater than the nominal, in cases that the sample size is very small and the values of both $\mathrm{k}_{1}$ and $\mathrm{k}_{2}$ are large, it appears to be smaller than it.

In conclusion, one may regard (9) as a very appealing technique for constructing lcls for $\mathrm{p}$, since it combines two fairly desirable features. These are the simplicity in its assessment and the proximity of its coverage to the nominal. 
Table 2. The observed coverage of $1 \mathrm{cl}$ (7) (top value) and

lcl (9) (bottom value) for confidence level 0.9

\begin{tabular}{||c|c|c|c|c||c|c|c|c|c||}
\hline $\begin{array}{c}\mathbf{k}_{\mathbf{1}} \\
\mathbf{k}_{\mathbf{2}}\end{array}$ & $\mathbf{n}=\mathbf{1 0}$ & $\mathbf{n}=\mathbf{3 0}$ & $\mathbf{n}=\mathbf{5 0}$ & $\mathbf{n}=\mathbf{1 0 0}$ & $\mathbf{k}_{\mathbf{1}}$ & $\mathbf{n}=\mathbf{1 0}$ & $\mathbf{n}=\mathbf{3 0}$ & $\mathbf{n}=\mathbf{5 0}$ & $\mathbf{n}=\mathbf{1 0 0}$ \\
$\mathbf{k}_{\mathbf{2}}$ & & & & & & \\
\hline 1,1 & 0.9623 & 0.9480 & 0.9407 & 0.9340 & 3,3 & 0.9510 & 0.9438 & 0.9357 & 0.9334 \\
& 0.9310 & 0.9215 & 0.9185 & 0.9167 & & 0.9104 & 0.9150 & 0.9116 & 0.9138 \\
\hline 1,2 & 0.9596 & 0.9537 & 0.9514 & 0.9538 & 3,4 & 0.9402 & 0.9380 & 0.9392 & 0.9424 \\
& 0.9300 & 0.9323 & 0.9342 & 0.9421 & & 0.8957 & 0.9088 & 0.9170 & 0.9274 \\
\hline 1,3 & 0.9522 & 0.9524 & 0.9478 & 0.9484 & 3,5 & 0.9358 & 0.9377 & 0.9415 & 0.9424 \\
& 0.9210 & 0.9334 & 0.9331 & 0.9372 & & 0.8907 & 0.9096 & 0.9207 & 0.9283 \\
\hline 1,4 & 0.9494 & 0.9470 & 0.9447 & 0.9444 & 3,6 & 0.9362 & 0.9378 & 0.9418 & 0.9402 \\
& 0.9195 & 0.9273 & 0.9298 & 0.9342 & & 0.8908 & 0.9102 & 0.9205 & 0.9254 \\
\hline 1,5 & 0.9484 & 0.9456 & 0.9478 & 0.9455 & 4,4 & 0.9431 & 0.9405 & 0.9383 & 0.9307 \\
& 0.9171 & 0.9274 & 0.9321 & 0.9354 & & 0.8998 & 0.9106 & 0.9130 & 0.9122 \\
\hline 1,6 & 0.9506 & 0.9464 & 0.9461 & 0.9435 & 4,5 & 0.9344 & 0.9350 & 0.9374 & 0.9364 \\
& 0.9186 & 0.9282 & 0.9315 & 0.9342 & & 0.8858 & 0.9039 & 0.9137 & 0.9187 \\
\hline 2,2 & 0.9558 & 0.9458 & 0.9409 & 0.9358 & 4,6 & 0.9319 & 0.9350 & 0.9372 & 0.9369 \\
& 0.9217 & 0.9200 & 0.9167 & 0.9155 & & 0.8820 & 0.9044 & 0.9132 & 0.9204 \\
\hline 2,3 & 0.9470 & 0.9499 & 0.9461 & 0.9508 & 5,5 & 0.9404 & 0.9388 & 0.9362 & 0.9248 \\
& 0.9070 & 0.9243 & 0.9257 & 0.9379 & & 0.8923 & 0.9089 & 0.9111 & 0.9066 \\
\hline 2,4 & 0.9420 & 0.9490 & 0.9475 & 0.9479 & 5,6 & 0.9304 & 0.9318 & 0.9294 & 0.9335 \\
& 0.9022 & 0.9258 & 0.9288 & 0.9346 & & 0.8766 & 0.8988 & 0.9023 & 0.9151 \\
\hline 2,5 & 0.9441 & 0.9492 & 0.9474 & 0.9476 & 6,6 & 0.9361 & 0.9330 & 0.9334 & 0.9286 \\
& 0.9040 & 0.9250 & 0.9269 & 0.9331 & & 0.8858 & 0.8991 & 0.9066 & 0.9087 \\
\hline 2,6 & 0.9438 & 0.9435 & 0.9510 & 0.9501 & & & & & \\
& 0.9042 & 0.9204 & 0.9320 & 0.9377 & & & & & \\
\hline
\end{tabular}

Table 3. The observed coverage of lcl (7) (top value) and

lcl (9) (bottom value) for confidence level 0.95

\begin{tabular}{||c|c|c|c|c||c|c|c|c|c||}
\hline $\begin{array}{c}\mathbf{k}_{\mathbf{1}} \\
\mathbf{k}_{\mathbf{2}}\end{array}$ & $\mathbf{n}=\mathbf{1 0}$ & $\mathbf{n}=\mathbf{3 0}$ & $\mathbf{n}=\mathbf{5 0}$ & $\mathbf{n}=\mathbf{1 0 0}$ & $\mathbf{k}_{\mathbf{1}}$ & $\mathbf{n}=\mathbf{1 0}$ & $\mathbf{n}=\mathbf{3 0}$ & $\mathbf{n}=\mathbf{5 0}$ & $\mathbf{n}=\mathbf{1 0 0}$ \\
\hline 1,1 & 0.9819 & 0.9736 & 0.9736 & 0.9692 & 3,3 & 0.9771 & 0.9748 & 0.9731 & 0.9688 \\
& 0.9663 & 0.9599 & 0.9610 & 0.9586 & & 0.9554 & 0.9602 & 0.9602 & 0.9588 \\
\hline 1,2 & 0.9774 & 0.9778 & 0.9761 & 0.9757 & 3,4 & 0.9704 & 0.9710 & 0.9731 & 0.9735 \\
& 0.9595 & 0.9664 & 0.9669 & 0.9684 & & 0.9447 & 0.9550 & 0.9612 & 0.9635 \\
\hline 1,3 & 0.9757 & 0.9724 & 0.9689 & 0.9697 & 3,5 & 0.9681 & 0.9717 & 0.9709 & 0.9723 \\
& 0.9566 & 0.9608 & 0.9598 & 0.9634 & & 0.9427 & 0.9550 & 0.9587 & 0.9637 \\
\hline 1,4 & 0.9738 & 0.9700 & 0.9700 & 0.9648 & 3,6 & 0.9674 & 0.9718 & 0.9714 & 0.9716 \\
& 0.9550 & 0.9584 & 0.9604 & 0.9590 & & 0.9414 & 0.9565 & 0.9589 & 0.9630 \\
\hline 1,5 & 0.9726 & 0.9690 & 0.9699 & 0.9650 & 4,4 & 0.9724 & 0.9718 & 0.9684 & 0.9689 \\
& 0.9529 & 0.9563 & 0.9611 & 0.9579 & & 0.9500 & 0.9556 & 0.9560 & 0.9588 \\
\hline 1,6 & 0.9742 & 0.9686 & 0.9685 & 0.9671 & 4,5 & 0.9688 & 0.9696 & 0.9694 & 0.9702 \\
& 0.9554 & 0.9560 & 0.9590 & 0.9602 & & 0.9419 & 0.9529 & 0.9564 & 0.9605 \\
\hline 2,2 & 0.9787 & 0.9748 & 0.9730 & 0.9691 & 4,6 & 0.9663 & 0.9709 & 0.9680 & 0.9692 \\
& 0.9597 & 0.9600 & 0.9607 & 0.9592 & & 0.9384 & 0.9539 & 0.9550 & 0.9602 \\
\hline 2,3 & 0.9730 & 0.9745 & 0.9740 & 0.9717 & 5,5 & 0.9725 & 0.9703 & 0.9692 & 0.9664 \\
& 0.9497 & 0.9600 & 0.9639 & 0.9628 & & 0.9455 & 0.9530 & 0.9561 & 0.9555 \\
\hline 2,4 & 0.9713 & 0.9750 & 0.9738 & 0.9734 & 5,6 & 0.9658 & 0.9654 & 0.9667 & 0.9683 \\
& 0.9472 & 0.9617 & 0.9627 & 0.9650 & & 0.9368 & 0.9470 & 0.9521 & 0.9583 \\
\hline 2,5 & 0.9712 & 0.9740 & 0.9738 & 0.9735 & 6,6 & 0.9708 & 0.9685 & 0.9656 & 0.9646 \\
& 0.9480 & 0.9602 & 0.9639 & 0.9646 & & 0.9428 & 0.9508 & 0.9500 & 0.9534 \\
\hline 2,6 & 0.9743 & 0.9723 & 0.9728 & 0.9719 & & & & & \\
& 0.9514 & 0.9590 & 0.9624 & 0.9642 & & & & & \\
\hline
\end{tabular}




\section{An Illustrative Example}

In order to illustrate the assessment of $1 \mathrm{cl} \mathrm{p}^{*}$ through (9), consider an example given by Wang and Lam (1996) in relation to their method. In that example, lcls were assessed for the true value of the proportion of conformance for a process with a lower specification limit equal to 68 and an upper specification limit equal to 78 . A random sample of size 30 was taken from this process yielding an average $\overline{\mathrm{X}}$ equal to 72.8 and a standard deviation $\mathrm{S}$ equal to 2 . For the above given specification limits and parameter estimates, the values of $\mathrm{K}_{1}$ and $\mathrm{K}_{2}$ are equal to 2.4 and 2.6, respectively.

As found by Wang and Lam (1996) the values of the 95\% lcls for p using formulae (3), (4) and (7), were $0.93798,0.9214$ and 0.9414 , respectively. For the new lcl, using the method proposed, we obtain from (9)

$$
\mathrm{C}_{1}=2.6\left(1+\frac{1}{30}\right) \sqrt{\frac{17.70837}{29}}=2.0994
$$

and

$$
\mathrm{C}_{2}=2.4\left(1+\frac{1}{30}\right) \sqrt{\frac{17.70837}{29}}=1.9379 .
$$

Therefore, the value of the lcl proposed is equal to

$$
\Phi\left(\frac{1}{\sqrt{30}}+2.0994\right)-\Phi\left(\frac{1}{\sqrt{30}}-1.9379\right)=0.94915 .
$$

This is clearly in line with the previously stated remarks on the behavior of lcl (9), since its value exceeds that obtained from (7).

\section{References}

Chou, Y-M. and Owen, D.B. (1984). One-Sided Confidence Regions on the Upper and Lower Tail Areas of the Normal Distribution. Journal of Quality Technology, 16(3), 150-158.

Kotz, S. and Johnson, N.L. (1993). Process Capability Indices, Chapman and Hall.

Kotz, S. and Johnson, N.L. (2001). Process Capability Indices - a Review, 1992-2000. Journal of Quality Technology. (To appear) 
Kotz, S. and Lovelace, C.R. (1998). Process Capability Indices in Theory and Practice. Arnold.

Owen, D.B. and Hua T.A. (1977). Tables of Confidence Limits on the Tail Area of the Normal Distribution. Communication in Statistics - Simulation and Computation, B6(3), 285-311.

Wald, A. and Wolfowitz, J. (1946). Tolerance Limits for a Normal Distribution. Annals of Mathematical Statistics, 14, 45-55.

Wang, C.M. and Lam, C.T. (1996). Confidence Limits for Proportion of Conformance. Journal of Quality Technology, 28(4), 439-445. 